\title{
Levels of Cognitive Control: A Functional Magnetic Resonance Imaging-Based Test of an RDoC Domain Across Bipolar Disorder and Schizophrenia
}

\author{
Jason Smucny*,', Tyler A Lesh', Keith Newton², Tara A Niendam', J Daniel Ragland' and Cameron S Carter' \\ 'Department of Psychiatry, University of California, Davis, Sacramento, CA, USA; ${ }^{2}$ College of Medicine, University of Cincinnati, Cincinnati, OH, USA
}

In recent years, the boundaries of psychopathology as defined by diagnostic categories have been criticized as inadequately 'carving nature at its joints' with respect to the neurobiology of major mental disorders. In 2010 the NIMH launched the Research Domain Criteria (RDoC) framework for understanding mental illnesses as brain circuit disorders that extend beyond DSM-defined diagnoses. In the present study we focus on cognitive dysfunction, a core feature of schizophrenia (SZ) and bipolar disorder (BPD), and use functional magnetic resonance imaging ( $\mathrm{fMRI}$ ) during a cognitive control (CC) task in recent onset patients to test the hypothesis that at a behavioral and underlying neural circuitry level these deficits exist on a continuum (as opposed to showing categorical differences) across the two disorders. In total, 53 healthy controls, 24 recent $(<1$ y) onset patients with BPD Type I with psychotic features, and 70 recent onset patients with SZ performed the AX-Continuous Performance Task while undergoing event-related fMRI at I.5 T. In addition to behavior task-associated response was examined in frontoparietal regions-of-interest. In an a priori contrast-based analysis, significant deficits across patient groups (vs controls) were observed on CC-associated performance as well as frontoparietal response. These analyses further revealed a continuum of deficits in which BPD showed intermediate levels of CC relative to controls and SZ. Poor CC was associated with poverty and disorganization symptoms across patient groups. These results support the hypothesis that CC dysfunction in BPD and SZ reflects a continuum of deficits that cuts across traditional, DSM-based classification. Implications for the neurobiology of these diseases are discussed.

Neuropsychopharmacology (2018) 43, 598-606; doi:I0.1038/npp.2017.233; published online I5 November 2017

\section{INTRODUCTION}

Although symptom-based diagnoses under DSM and ICD guidelines have proven invaluable both in the clinic and for research, it has been recognized by the National Institutes of Mental Health (NIMH) that this 'Kraepelinian' nosological framework may not accurately capture underlying mechanisms that underlie psychiatric dysfunction in that these processes may cross diagnostic boundaries (Insel et al, 2010). For example, studies have observed shared biological and psychological features across bipolar disorder (BPD) and schizophrenia (SZ) diagnoses despite the fact that these disorders are classified by the DSM as distinct entities. These shared features include varying degrees of common genetic susceptibility loci (Craddock et al, 2006), changes in neuronal structure and function (Konopaske et al, 2014; McIntosh et al, 2009; O'Donoghue et al, 2017; Whalley et al, 2012), and cognitive disability (Bora and Pantelis, 2015). Psychosis is another particularly striking feature that is often shared between SZ and BPD (Type I), and consequently the same antipsychotics are often prescribed to treat the two

*Correspondence: Dr J Smucny, Imaging Research Center, University of California, Davis, 470I X Street, Sacramento, CA 95817, USA. Tel: + I 9|6-734-3230, Fax: + I- 9|6-734-8750, E-mail: jsmucny@ucdavis.edu Received 8 July 2017; revised 25 August 2017; accepted 21 September 2017; accepted article preview online 26 September 2017 diseases (Pearlson, 2015; Stahl, 2014; Tamminga et al, 2014). The degree to which these disorders may lie along a single continuum and consequently be manifestations of shared biological processes is therefore a major research question in psychiatry.

Cognitive deficits in BPD and SZ (and their associated neuronal mechanisms) are particularly important areas for further research given that no FDA-approved treatment exists for these deficits even though they predict functional outcome in both diseases (Green, 2006). Interestingly, one aspect of cognition in particular, cognitive control (CC), may be an especially intriguing potential target due the theory that it is a domain-generalizable process, such that a loss of CC may help explain dysfunction across a wide range of cognitive domains (Lesh et al, 2011; Niendam et al, 2012), as well other symptoms (Cole et al, 2014). Broadly speaking, CC refers to processes by which behavior can adapt from moment to moment based on current goals even in the face of stimuli from the environment that elicit habitual responses (Lesh et al, 2011). CC in this context should not be confused with the 'cognitive control' biotype isolated by cluster analysis by the Bipolar Schizophrenia Network on Intermediate Phenotypes (B-SNIP) consortium (Clementz et al, 2016). In the present study, CC refers to overarching cognitive processes and not task performance-defined patient groups. CC encapsulates a range of functions, 
including goal maintenance and adaptive or reactive control (Lesh et al, 2011). Of these, the Cognitive Neuroscience Treatment Research to Improve Cognition in Schizophrenia (CNTRICS; http://cntrics.ucdavis.edu) initiative has identified goal maintenance as an important target for treatment development in SZ (Carter et al, 2012).

As suggested by CNTRICS, the AX version of the continuous performance task (AX-CPT) has become a standard method of examining the mechanisms of goal maintenance of CC in SZ. In the AX-CPT, subjects are presented with a series of cues and probes and are instructed to respond to a probe only if it was preceded by the target ('A') cue. Goal maintenance is measured by comparing neuronal response to A (low control) cues vs non-A (high control, 'B') cues. Previous AX-CPT studies have observed impaired goal maintenance, as well as diminished recruitment of the dorsolateral prefrontal cortex (DLPFC) and superior parietal cortex (SPC), in SZ (Lesh et al, 2013, 2015; Niendam et al, 2014). In regard to BPD, a previous AX-CPT behavioral study reported that BPD patients performed (AX errors) intermediately between controls and SZ patients, suggestive of a continuum of deficits across disorders (Brambilla et al, 2007). A more recent behavioral study in a much larger sample using the dot probe expectancy task (DPX; an AX-CPT variant) found the same pattern of CC deficits driven by significant differences between HC, BPD, and SZ groups (Reilly et al, 2017).

The goal of the present study is to test the hypothesis that deficits that underlie goal maintenance across BPD and SZ may be described as a continuum of dysfunction that transcends DSM-based classification. Specifically, we hypothesize (1) the presence of impaired goal maintenance of CC in patients ( $v s$ controls), and (2) while SZ and BPD would show the same pattern of cognitive and functional neural circuitry deficits, this effect would vary in severity across the recent onset groups, with a significant linear trend reflecting $\mathrm{CC}$ in $\mathrm{BPD}$ that lies between controls and SZ patients. This pattern would be consistent with previous AXCPT behavioral results (Brambilla et al, 2007) cognitive meta-analyses (Bora and Pantelis, 2015; Reichenberg and Harvey, 2007; Robinson et al, 2006), and several findings from the Bipolar-Schizophrenia Network on Intermediate Phenotypes (B-SNIP) consortium (Hill et al, 2013; Ivleva et al, 2016; Reilly et al, 2017; Sheffield et al, 2017). As we were interested in common phenomenological endpoints (eg, psychosis, possibly suggestive of shared etiologies) we restricted our analysis to BPD Type I patients (who presented with psychotic features). We also considered this BPD variant to be clinically most 'similar' to SZ (rather than BPD Type II, for which depressive features are most prevalent) due to the increased genetic similarity between $\mathrm{SZ}$ and BPD relative to SZ and major depressive disorder (Cross-Disorder Group of the Psychiatric Genomics et al, 2013).

\section{MATERIALS AND METHODS}

\section{Subjects}

In total 147 subjects (ages 16-30) participated in this study70 patients with either SZ $(n=58)$, schizophreniform $(n=6)$ or schizoaffective $(n=6)$ disorder, 24 patients with BPD
Type I with psychotic features, and 53 healthy control subjects. Of the present sample, data from 43 controls and 32 SZ patients were included in a previous AX-CPT study that focused exclusively on SZ (Lesh et al, 2013), from 16 controls and 16 patients in another AX-CPT study in SZ (Yoon et al, 2008), and from 15 controls and 24 patients in a third AXCPT study in SZ and high-risk subjects (Niendam et al, 2014). All patients were recruited as outpatients from the University of California, Davis (UCD) Early Diagnosis and Preventive Treatment (of Psychosis) (EDAPT) research clinic, with their first psychotic episode occurring within 1 year of study. Patients were assessed with the Structured Clinical Interview for DSM-IV-TR (SCID) (First et al, 2002). Patients were followed longitudinally and diagnoses were confirmed by SCID and consensus conference 12 months after ascertainment. Patients were excluded for a diagnosis of major medical or neurological illness, head trauma, substance abuse in the previous 3 months (as well as a positive urinalysis on the day of scanning), Weschler Abbreviated Scale of Intelligence-2 score (WASI) (Weschler, 1999) score $<70$, and magnetic resonance imaging (MRI) exclusion criteria (eg, claustrophobia, metal in the body). Control subjects were excluded for all of the above as well as a history of Axis I mental illness or first-degree family history of psychosis. All participants provided written informed consent and were compensated for participation. The UCD Institutional Review Board approved the study. CPZ equivalent antipsychotic doses were calculated using published guidelines for conventional (American Psychiatric Association, 1997) and atypical (Woods, 2003) antipsychotics. Consistent with prior work (Barch et al, 2003), three core symptom dimensions were calculated. 'Poverty' combined emotional withdrawal, motor retardation, and blunted affect from the Brief Psychiatric Rating Scale (BPRS) (Ventura et al, 1993) with anhedonia/asociality, avolition/ apathy, alogia, and affective flattening from the Scale for the Assessment of Negative Symptoms (SANS) (Andreasen, 1984a). 'Disorganization' combined conceptual disorganization, mannerisms and posturing, and disorientation scores from the BPRS with attention score from the SANS as well as positive formal thought disorder and bizarre behavior scores from the Scale for the Assessment of Positive Symptoms (SAPS) (Andreasen, 1984b). 'Reality distortion' combined grandiosity, suspiciousness, hallucinations, and unusual thought content from the BPRS with hallucinations and delusions from the SAPS (Barch et al, 2003). It should be noted that these symptom dimensions have variable longterm stability (Arndt et al, 1995; Marengo et al, 2000) and we must therefore remain agnostic as to the degree of state or trait-like clinical status of the present sample.

\section{Task Description}

The AX-CPT and associated task parameters have been described in detail elsewhere (Cohen et al, 1999; Lesh et al, 2013). Briefly, participants are presented with a series of cues and probes and are instructed to make a target response (pressing a button with the index finger) to the probe letter ' $\mathrm{X}$ ' only if it was preceded by the cue letter 'A'. All cues and nontarget probes require nontarget responses (pressing a button with the middle finger). Target sequence trials (ie, 'AX' trials) are frequent (70\% occurrence) and set up a 
prepotent tendency to make a target response when the probe letter X occurs. As a result, nontarget sequence trials in which any non-A cue (collectively called ' $B$ ' cues) is presented and followed by a probe letter X (ie, 'BX' trials) require the most cognitive control. $\mathrm{AY}$ and $\mathrm{BY}$ trials are included to test for generalized deficits and represent an internal control. Cues and probes were presented for $500 \mathrm{~ms}$, with a $3500 \mathrm{~ms}$ interval between cue and probe and a $9500 \mathrm{~ms}$ intertrial interval. Trial distribution was 70\% AX, $10 \%$ AY, $12.5 \%$ BX, 7.5\% BY. The task consisted of 4 runs of 40 trials for a total of 160 trials and a total time of $37 \mathrm{~min}$ $20 \mathrm{~s}$. The task was presented using EPrime2 software (Psychology Software Tools, Inc.).

The primary behavioral outcome measure of interest was d-prime context, calculated as a function of AX hits minus BX false alarms (Cohen et al, 1999). AX-CPT d-prime context has been shown to have moderate to good reliability, with intraclass coefficients between 0.73 and 0.78 depending on delay time and 0.81 when collapsing across delay time in a pool of healthy control subjects, psychotic non-SZ (primarily mood disorder) patients, and SZ patients (Barch et al, 2009). D-prime context (z-score) was analyzed by ANCOVA with group and gender as between-subjects factors (due to gender imbalance between groups). As part of the ANCOVA, two $a$ priori contrasts were performed. The first contrast compared healthy controls to patients (as a whole) to test the hypothesis that patients would show overall deficits in $\mathrm{d}$-prime context. The second contrast was a polynomial linear trend analysis $\{$ controls $>\mathrm{BPD}>\mathrm{SZ}$ \} to test the hypothesis that BPD patients would show intermediate goal maintenance-related performance deficits relative to SZ patients, indicative of a continuum of deficits. The null hypothesis of this orthogonal contrast is a zero slope. To more completely characterize group effects, auxiliary pairwise post-hoc tests were also performed comparing d-prime context between each group (eg, SZ vs BPD patients). Significance for post-hoc tests was set to $\mathrm{p}<0.05$; $p$ values between 0.05 and 0.10 were considered trend-level.

An exploratory analysis of auxiliary performance metrics (accuracy and reaction time) was performed by repeated measures ANCOVA, with trial type (AX/AY/BX/BY) as a within-subjects factor and diagnosis and gender as betweensubjects factors. Contrasts were not performed as part of this analysis as we did not have a priori hypotheses regarding these measures.

\section{fMRI Scanning}

Studies were performed with at $1.5 \mathrm{~T}$ GE Signa MR system using a standard head coil. Functional images were acquired with a gradient-echo $\mathrm{T} 2 *$ Blood Oxygenation Level Dependent (BOLD) contrast technique, with $\mathrm{TR}=2000 \mathrm{~ms}$, $\mathrm{TE}=40 \mathrm{~ms}, \mathrm{FOV}=220 \mathrm{~mm}^{2}$, flip angle $=90^{\circ}$, and 24 axial slices (contiguous and interleaved, $4 \mathrm{~mm}$ thick, with a $3.4 \mathrm{~mm}^{2}$ in-plane resolution).

\section{fMRI Preprocessing}

fMR images were preprocessed using SPM8 (Wellcome Department of Imaging Neuroscience, London). Briefly, images were slice-timing corrected, realigned, normalized to the Montreal Neurological Institute template using a rigid- body transformation followed by non-linear warping, and smoothed with an $8 \mathrm{~mm}$ FWHM Gaussian kernel. All individual fMRI runs had $<4 \mathrm{~mm}$ of translational withinrun movement, $<3$ degrees of rotational within-run movement, and $<0.45 \mathrm{~mm}$ of average framewise displacement (calculated using the fsl_motion_outliers tool) (https://fsl. fmrib.ox.ac.uk/fsl/fslwiki/FSLMotionOutliers). All subjects had at least two fMRI runs surviving these criteria.

\section{Region-of-Interest Analysis of BOLD Response}

First-level effects were modeled with a double-gamma function, without temporal derivatives, using the general linear model in SPM8. 'Task-associated' (B>A Cue) contrast images (parameter estimates) were generated for each subject. The $\mathrm{B}>\mathrm{A}$ contrast measures response under conditions of high $v s$ low cognitive control (Lesh et al, 2015; Lesh et al, 2013). Images were motion-corrected using rigid-body motion parameters as single-subject regressors. All trial types were modeled (AX/AY/BX/BY) and only correct responses were used to create first-level images consistent with previous studies (Lesh et al, 2013, 2015).

Using first-level images, a priori hypotheses of taskassociated response were examined in two bilateral, $4 \mathrm{~mm}$ radius spherical region-of-interests (ROIs): the DLPFC and SPC. As ROI size was chosen arbitrarily, the analysis was repeated for 6 and $8 \mathrm{~mm}$ radii. The DLPFC ROI was taken from a previous study from an independent Stroop task data set (MacDonald et al, 2000). The SPC ROI was taken from a previous meta-analysis of non n-back studies of executive function in SZ (Minzenberg et al, 2009). Mean taskassociated response ( $\mathrm{z}$-score) in these ROIs was extracted using the Marsbar toolbox (Brett et al, 2002) and examined by ANCOVA with diagnosis and gender as between-subjects factors. As was the case for behavioral data, two a priori contrasts were performed. The first contrast compared healthy controls to patients (as a whole) to test the hypothesis that patients would show overall deficits in task-associated activity within these ROIs. The second contrast was a polynomial linear trend analysis \{controls $>$ $\mathrm{BPD}>\mathrm{SZ}$ \} to test the hypothesis that BPD patients would show mitigated BOLD response deficits relative to SZ patients, indicative of a continuum of dysfunction. As described for d-prime context, auxiliary pairwise post-hoc tests were also performed comparing ROI BOLD response between each group.

\section{Whole-Brain Analysis}

In addition to ROI analysis, an exploratory whole-brain analysis was conducted by ANOVA using the factors and contrasts defined above. Significance was set to voxelwise $p<0.001$ (uncorrected), clusterwise $p<0.05$ (FWE-corrected for multiple comparisons).

\section{Behavioral and Clinical Correlates}

Associations between CC-related (d-prime context, taskassociated DLPFC and SPC ROI activation) and clinical (poverty, disorganization, reality distortion) measures were examined using Spearman's rho correlation coefficients as not all data were normally distributed. An additional analysis 
was conducted examining the relationship between antipsychotic dose and CC.

Correlates were analyzed across groups, as we were interested in associations that crossed diagnostic categories consistent with the $\mathrm{RDoC}$ framework. To ensure that correlations were not driven by group differences, associations were adjusted for the effect of group by calculating normalized residuals (zscores) from the linear regression of group to each measure prior to correlation analyses. As these analyses were exploratory, significance was set to $p<0.05$ (uncorrected). $P$ values between 0.05 and 0.10 were considered trend-level.

\section{RESULTS}

\section{Demographics}

Demographic and clinical information for participants are shown in Table 1. Groups did not differ significantly by age or handedness but did differ by gender. Gender was therefore included as a factor in all between-group analyses. Results from other demographic and clinical between-group comparisons are provided in Table 1.

\section{Behavioral Results}

For all analyses, subjects with d-prime context or ROI BOLD response outliers $>4 \mathrm{SD}$ below the group mean were censored from analysis (2 SZ patients). Notably, inclusion of these outliers did not appreciably alter group results (slightly increased significance for contrasts of interest).

Performance metrics for the AX-CPT for each group are presented (as a dot plot) in Figure 1 and Supplementary Table 1. All subjects performed adequately on the task, suggesting they understood the instructions and were performing the task ('adequately' was defined as accuracy greater than or equal to $44 \%$ on $\mathrm{AX}$ trials and $50 \%$ on BY trials) (Henderson et al, 2012). For d-prime context, the overall model (including gender) was significant $(F(5,139)=6.04, p<0.001)$ as was the main effect of group $(F(2,139)=10.80, p<0.001)$. No main effect of gender or gender $\mathrm{X}$ diagnosis interaction was observed. The a priori contrast testing the hypothesis of deficits in patients (controls $>$ patients) for d-prime context was significant (contrast estimate $=-0.63,95 \% \mathrm{CI}=\{-0.98$ to -0.28$\}$, $p=0.001)$. The a priori contrast testing the hypothesis of a linear trend (controls $>\mathrm{BPD}>\mathrm{SZ}$ ) was also significant (contrast estimate $=-0.53,95 \% \mathrm{CI}=\{-0.75$ to -0.30$\}$, $p<0.001)$. Pairwise post-hoc tests revealed significant

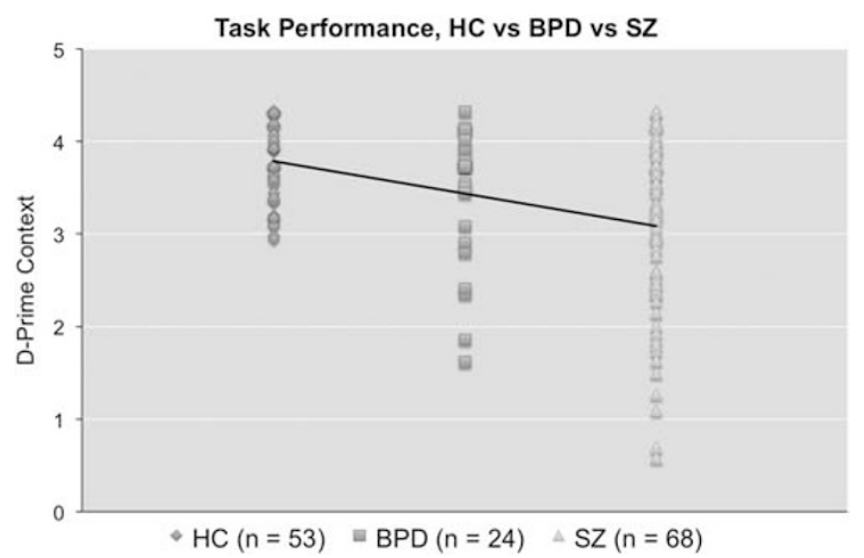

Figure I Plot of individual d-prime context scores for each diagnostic group on the AX-CPT. Straight line represents the slope of the linear contrast $\mathrm{HC}>\mathrm{BPD}>\mathrm{SZ}$.

Table I Demographic and Clinical Information for Participants

\begin{tabular}{|c|c|c|c|c|c|c|c|}
\hline & $\mathrm{HC}$ & SD & BPD & SD & SZ & SD & $\mathbf{F}, \mathbf{t}$ or $\chi^{2}(\mathbf{p})$ \\
\hline Age & 21.06 & 2.83 & 22.55 & 2.99 & 21.03 & 3.02 & $2.62(0.076)$ \\
\hline Gender (M/F) & $31 / 22$ & & $15 / 9$ & & $59 / 11$ & & |2.| $(0.002)^{\mathrm{a}}$ \\
\hline Handedness (R/L) & $48 / 5$ & & $22 / 2$ & & $68 / 2$ & & $1.76(0.42)$ \\
\hline Education Level (Years) & 14.13 & 1.93 & 13.04 & 1.43 & 12.53 & 1.76 & $12.3(0.00 \mid)^{\mathrm{a}}$ \\
\hline Parental Education Level & $|4.5|$ & 2.39 & 14.22 & 2.30 & 14.89 & 2.60 & $0.75(0.48)$ \\
\hline Antipsychotics (CPZ Equivalent Dose, Mg/Day) & & & 202.23 & 108.99 & 257.64 & 214.06 & $1.35(0.19)$ \\
\hline Other Mood Stabilizer (Valproate/Lithium/None) & & & $6 / 5 / 13$ & & n/a & & \\
\hline IQ (WASI) & 113.90 & 10.46 & 105.17 & 12.93 & 103.07 & 12.69 & $11.7(<0.00 \mid)^{\mathrm{a}}$ \\
\hline \multicolumn{8}{|l|}{ Core Symptom Dimensions } \\
\hline CDS Total ${ }^{a}$ & & & 3.05 & 3.85 & 1.28 & 1.75 & $1.89(0.072)$ \\
\hline
\end{tabular}

Abbreviations: CDS, Calgary Depression Scale; CPZ, chlorpromazine; GAF, Global Assessment of Function; HAM-D, Hamilton Depression Rating Scale; SD, standard deviation; WASI, Wechsler Abbreviated Scale of Intelligence; YMRS, Youth Mania Rating Scale.

${ }^{a}$ Calgary Depression Scale scores were unavailable for 5 BPD and 4 I SZ patients.

$T$ values are provided for parametric comparisons between two groups; other wise $\mathrm{F}$ or $\chi^{2}$ values are provided. $* p<0.05$. 
differences between controls and SZ patients $(p<0.001)$, as well as SZ and BPD patients $(p=0.044)$. A trend-level difference was observed between controls and BPD patients $(p=0.067)$.

For auxiliary behavioral measures, significant diagnosis (controls $v s \mathrm{SZ} v s \mathrm{BPD}) \mathrm{X}$ trial type (AX vs $\mathrm{AY} v s \mathrm{BX} v s \mathrm{BY}$ ) interactions were observed for accuracy (Wilks' $\lambda=0.88, \mathrm{~F}$ $(6,274)=3.16, p=0.005)$ and reaction time (Wilks' $\lambda=0.87$, $\mathrm{F}(6,274)=2.96, p=0.008$; Supplementary Table 1). No main effects of gender or gender $\mathrm{X}$ diagnosis interactions were observed for either accuracy or reaction time.

\section{fMRI Results: ROI Analysis}

Statistical parametric maps of task-associated response and dot plots showing distribution of response for each group and clusters from the linear contrast (control $>$ BPD $>$ SZ) are shown in Figure 2. Group means and standard deviations are presented in Supplementary Table 2. For task-associated response of the DLPFC, the overall model (including gender) was significant $(\mathrm{F}(5,139)=2.77, p=0.020)$ as was the main effect of group $(\mathrm{F}(2,139)=4.27, p=0.016)$. No effect of gender or gender $\mathrm{X}$ group interaction was observed. The a priori contrast testing the hypothesis of DLPFC BOLD response deficits in patients (controls $>$ patients) was significant (contrast estimate $=-0.54,95 \%$ C.I. $=\{-0.91$ to -0.17$\}$, $p=0.004)$. The a priori contrast testing the hypothesis of a linear trend (controls $>\mathrm{BPD}>\mathrm{SZ}$ ) was also significant (contrast estimate $=-0.43,95 \%$ C.I. $=\{-0.70$ to $-0.16\}, \quad p=0.002)$. Pairwise post-hoc tests revealed significant differences between controls and SZ patients $(p=0.002)$, as well as a trend-level difference between controls and BPD patients $(p=0.063)$. No trend-level or greater difference was observed between SZ and BPD patients.

For the bilateral SPC ROI, although the overall model (including gender) did not reach significance $(F(5,139)=1.85$, $p=0.11$ ) the main effect of group was significant ( $F$ $(2,139)=3.66, p=0.028)$ and no effect of gender or gender $\mathrm{X}$ group interaction was observed. The $a$ priori contrast testing the hypothesis of SPC BOLD response deficits in patients (controls $>$ patients) was significant (contrast estimate $=$ $-0.49,95 \%$ C.I. $=\{-0.86$ to -0.11$\}, p=0.011$ ). The a priori contrast testing the hypothesis of a linear trend (controls $>\mathrm{BPD}>\mathrm{SZ}$ ) was also significant (contrast estimate $=$ $-0.42,95 \%$ C.I. $=\{-0.69$ to -0.14$\}, p=0.003$ ). Pairwise posthoc tests revealed significant differences between controls and SZ patients $(p=0.005)$, a trend-level difference between controls and BPD patients $(p=0.058)$, and no trend-level or greater difference between SZ and BPD patients.

Increasing ROI size to a $6 \mathrm{~mm}$ or $8 \mathrm{~mm}$ radius did not appreciably alter contrast results (Supplementary Table 2).

\section{fMRI Results: Whole Brain Analysis}

The a priori contrast testing the hypothesis of BOLD response deficits in patients (controls $>$ patients) yielded significant clusters in the left DLPFC, right DLPFC, left SPC, right SPC, and anterior cingulate (ACC) (Supplementary Table 3a; Supplementary Figure 1a). The a priori contrast testing the hypothesis of a linear trend (controls $>$ BPD $>$ SZ) yielded significant clusters in the left DLPFC, right DLPFC, and right SPC (Supplementary Table 3b).

As a significant ACC cluster was observed using the control $>$ patient contrast but not the linear trend contrast (control $>\mathrm{BPD}>\mathrm{SZ}$ ), the nature of the control $>$ patient effect was further explored by extracting BOLD response from a $1 \mathrm{~mm}$ radius sphere centered at the cluster peak $(x, y$, $z=8,20,48)$. This analysis showed the ACC cluster was driven by a different pattern in which SZ patients showed intermediate deficits between controls and BPD patients (Supplementary Figure 1b).

\section{Clinical Correlates}

After controlling for group differences, across patient groups lower d-prime context was associated with higher disorganization symptoms ( $r h o=-0.21, p=0.048$; Figure 3, top). A trend-level relationship was also observed between lower $\mathrm{d}$-prime context and higher poverty symptoms ( $\mathrm{rho}=-0.20$, $p=0.059$; Figure 3, bottom). No relationship was observed between d-prime context and reality distortion symptoms. No significant relationships were observed between taskassociated BOLD response and symptoms. No relationships were observed with antipsychotic dose.

\section{DISCUSSION}

In agreement with our hypothesis, significant group effects were observed on performance on the AX-CPT task as well as goal maintenance-associated response in the DLPFC and SPC. A priori contrasts supported the hypothesis that recent onset patients (as a whole) showed deficits in d-prime context and CC-associated frontoparietal BOLD response in these regions. Furthermore, in support of our prediction of a continuum of dysfunction (with more severe deficits in SZ), linear contrasts of CC-related behavior within predefined frontoparietal (DLPFC and SPC) ROIs reflected a pattern of deficits in BPD patients that was intermediate between controls and SZ patients. Exploratory whole-brain analysis yielded an additional significant cluster in the ACC using the contrast controls $>$ patients, although this cluster was driven by the pattern $\mathrm{HC}>\mathrm{SZ}>\mathrm{BPD}$. Significant or trend-level associations were observed between poverty and disorganization symptoms and poor task performance across BPD and SZ patient groups, suggesting these relationships may transcend diagnostic categories. Importantly, these findings enrich those from previous behavioral AX-CPT studies (Brambilla et al, 2007; Reilly et al, 2017) by suggesting that the previously reported intermediate deficits in BPD (relative to SZ and healthy subjects) are also associated with reductions in frontoparietal activation.

To place these findings in context, it may be helpful to consider a recent commentary by Cole et al (2014). Cole and colleagues argue that a dysfunctional frontoparietal control system may be explicitly tied to symptomatology. Specifically, the authors suggest that in psychiatric disease, the cognitive control system is less able to regulate other functional neural systems in a goal-directed manner, leading to behavioral changes that manifest themselves as diagnosable symptoms. For example, behavioral disorganization, for which relationships to CC processes have been shown 

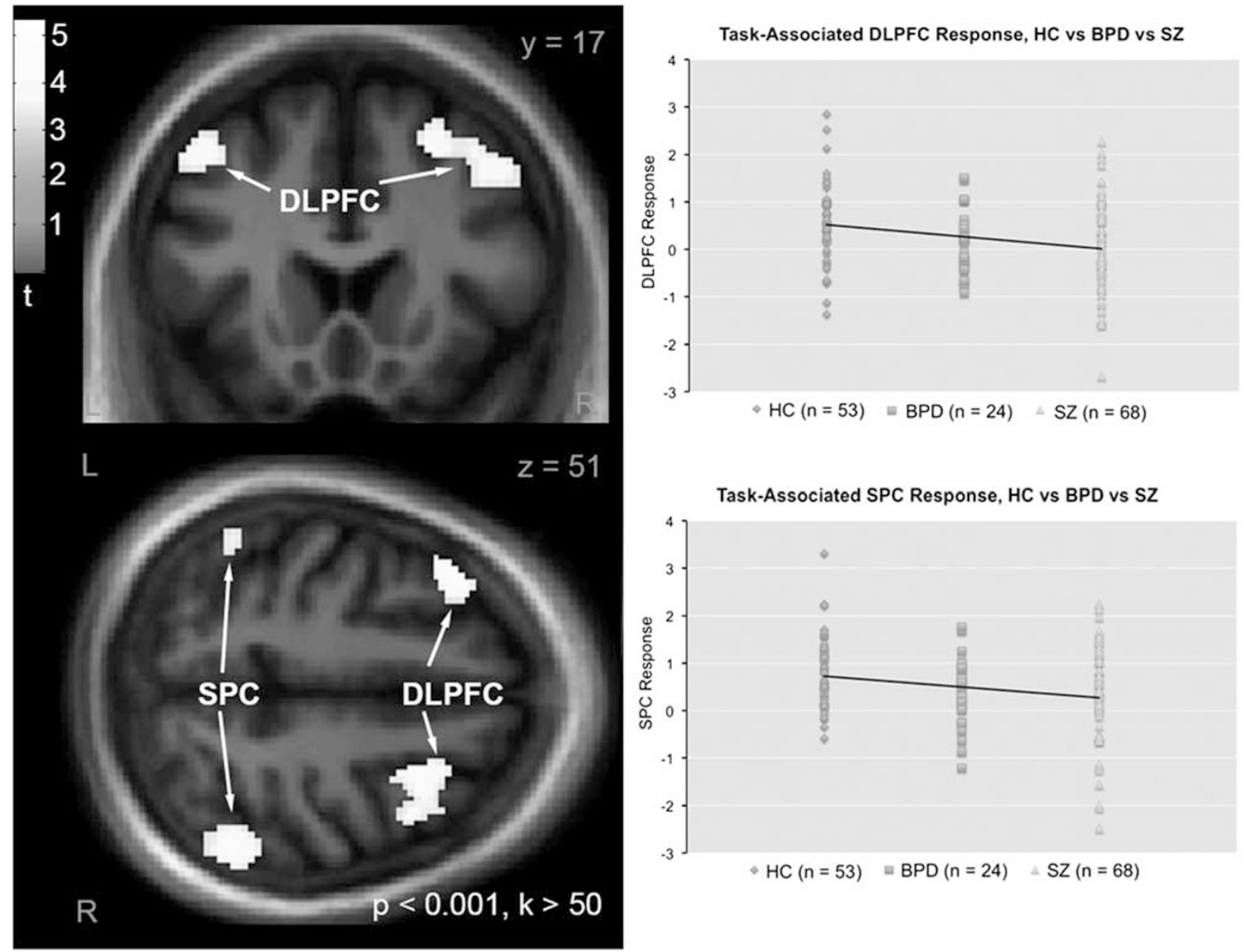

Task-Associated SPC Response, HC vs BPD vs SZ

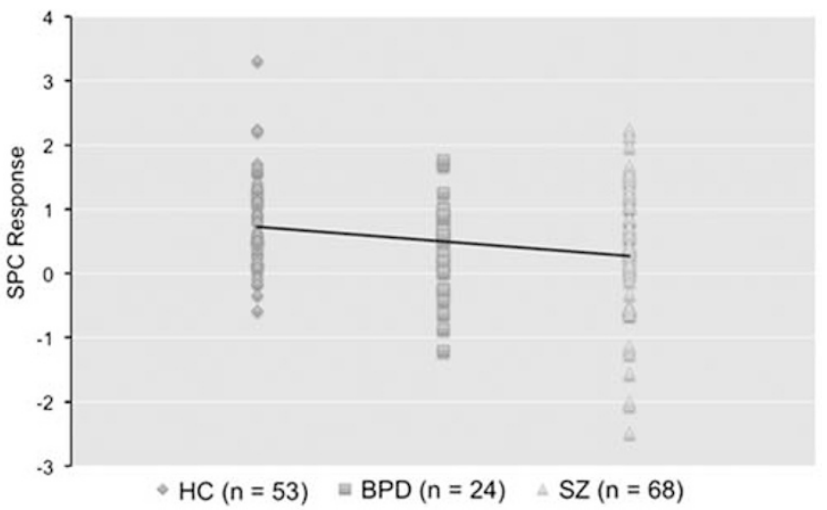

Figure 2 Left: Statistical parametric t map of task-associated BOLD response demonstrating significant linear trends (HC $>B P D>S Z$ directional contrast) on DLPFC and SPC signal. Map masked by a whole brain gray matter mask and thresholded at whole-brain voxelwise $p<0.00 \mathrm{I}, k>50$ voxels for visualization purposes. Map displayed in the neurologic convention ( $R$ on R). Top Right: Plot of individual task-associated BOLD response (beta weights) within the bilateral DLPFC ROI for each diagnostic group. Straight line represents the slope of the linear contrast HC>BPD >SZ. Bottom Right: Plot of task-associated BOLD response (beta weights) within the bilateral SPC ROI for each diagnostic group. Straight line represents the slope of the linear contrast HC > BPD > SZ.

previously (Yoon et al, 2008) and in this study, may be an emerging consequence of the inability of the CC system to guide goal-directed behavior. Within this framework the reverse relationship may also exist in that in psychiatric disease, the CC system needs to expend resources in an attempt to manage symptoms and consequently may be less able to be recruited during CC tasks. Importantly, within this framework loss of frontoparietal-directed CC is expected to manifest itself across diagnostic boundaries in a manner consistent with NIMH Research Domain Criteria (RDoC) (Insel et al, 2010). Although the direction of potential causal relationships between CC and clinical indicators is beyond the scope of this study, our findings suggest that CC abnormalities exist across diagnostic spectra and are correlated with some aspects of symptomatology.

Significant group differences in task-associated response were observed in both the DLPFC and SPC, suggesting that different subsystems of the CC network may be affected across disorders. Previous work has demonstrated that the DLPFC is involved in maintaining goal representations (Rougier et al, 2005), whereas the SPC is important for top- down control of visual attention and representing stimulusresponse mappings (Bunge et al, 2003). The present results suggest, therefore, that both processes are affected in BPD Type I (with psychotic features) and SZ. The observed pattern of deficits is also consistent with previous findings in BPD and SZ during working memory (Brandt et al, 2014; Hamilton et al, 2009), verbal fluency (Costafreda et al, 2011), and during the 'resting' state (absence of a task) (Baker et al, 2014). At odds with these findings, however, is a previous report during a sentence completion task that showed DLFPC hypoactivation in SZ patients but hyperactivation in BPD (McIntosh et al, 2008). While differences in clinical characteristics between that study and the present work (eg disparity of $\sim 8$ in mean YMRS scores of BPD groups) could explain these discrepant results, this discordancy suggests that the observed patterns of deficits observed in this study may not generalize across all cognitive domains or tasks. Prefrontal tasks that involve emotional cues or recruit emotion-related circuits, for example, may evoke hyperactive responses in BPD relative to other psychiatric groups or control subjects (Strakowski et al, 2011). Future studies may 


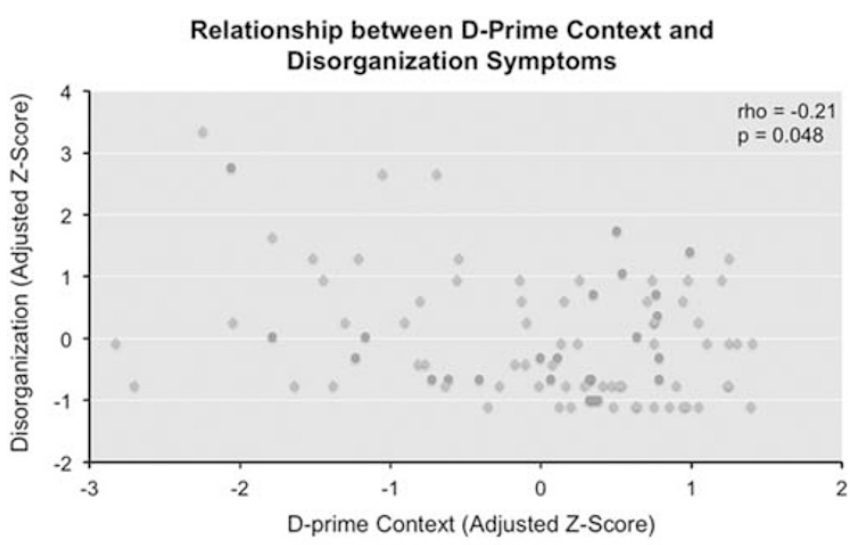

$\cdot \mathrm{BPD} \cdot \mathrm{SZ}$

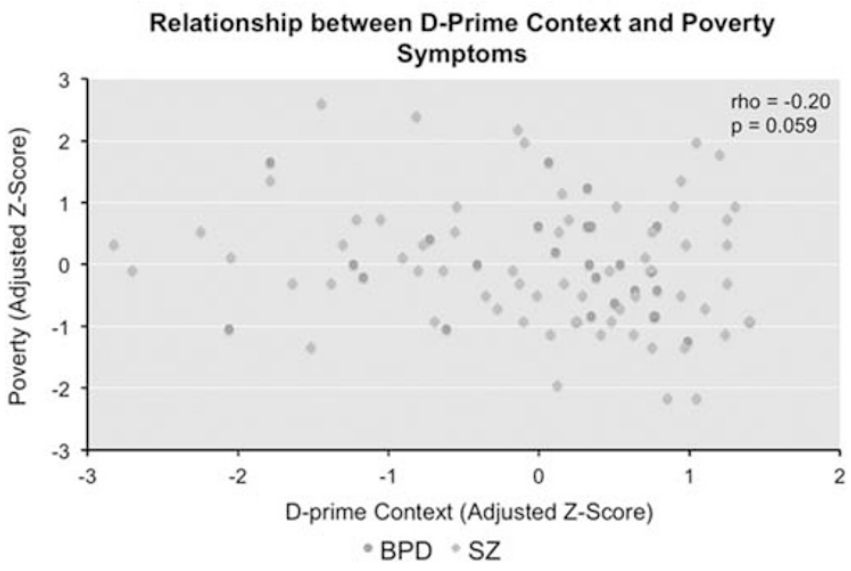

Figure 3 Associations between lower d-prime context (group-adjusted $z$-scores) and disorganization (group-adjusted z-scores) (top) and poverty (group-adjusted z-scores) (bottom) across patient groups. Subjects are color-coded by group for visualization purposes.

examine the effect of emotional distractors or cues on taskassociated response during CC performance in order to examine this possibility, as well as the generalizability of the findings to other related clinical groups (eg, BPD Type II or those without a history of psychotic features).

Exploratory whole-brain analysis using the controls $>$ patients (as a whole) contrast yielded an additional significant cluster in the ACC. Interestingly, this cluster appeared to be driven by a different pattern in which SZ patients showed deficits in-between that of controls and BPD. Consistent with the finding of a functional ACC deficit in psychosis, a 2009 meta-analysis of 41 studies of executive function showed loss of ACC recruitment in SZ (Minzenberg et al, 2009). Reduced ACC activity during a CC task has been observed in BPD as well (eg, Gruber et al, 2017). As the ACC is thought to be involved with performance monitoring and (not goal maintenance) of CC (Lesh et al, 2011) and was also not previously recruited during similar CC tasks (Lopez-Garcia et al, 2016), we did not hypothesize to observe differential task-associated response in this region in this study and this result therefore requires follow-up using an independent dataset. Nonetheless, this preliminary finding suggests that dysfunctional brain circuitry in SZ and BPD may not all show the same pattern of deficits and consequently BPD should be not be simply thought of as a generalized 'less

severe' form of SZ. Rather, the HC $>$ BPD $>$ SZ functional pattern may be specific to the frontoparietal CC network previously implicated in SZ (Cole et al, 2014).

The results of this study do not necessarily suggest that the pattern of cognitive deficits in SZ and BP is specific to goal maintenance and CC. Indeed, the B-SNIP consortium has reported the same pattern of deficits/abnormalities in BPD and SZ using a generalized neurocognitive battery (Hill et al, 2013). In that study the same pattern of deficits persisted across all cognitive domains tested (Hill et al, 2013). Nonetheless, as abnormal CC processes are known to contribute to cognitive deficits across a range of domains (Lesh et al, 2011; Niendam et al, 2012), it is likely that the results observed in the B-SNIP study may be driven by the CC deficits reported here. Related to this point, another B-SNIP report found that generalized cognitive deficits accounted for reduced goal maintenance in BPD and SZ during the DPX (Reilly et al, 2017), although the reverse relationship (the influence of goal maintenance on general cognition) was not examined.

A potential limitation of the present study is that differences between patients and controls may be confounded by antipsychotic treatment. Although no relationships were observed between antipsychotic dose and any behavioral or fMRI-related measure in patients (after correcting for group differences), the impact of antipsychotic medication on the observed linear trends cannot be fully ascertained as the control group was not taking antipsychotics. This question may be more thoroughly addressed in a future case-control or longitudinal CC-related fMRI study designed to specifically examine antipsychotic dose-response curves. As noted by Pearlson (2015), symptoms (particularly positive symptoms) often respond to the same medication regardless of diagnosis, and it is possible that any treatment designed to target CC deficits will show efficacy across categorical distinctions.

BPD and SZ share neurochemical and neuroanatomical features in cortical areas that may help explain common deficits in CC processes. Both diseases are associated with abnormal expression of GABAergic (Volk et al, 2016), cholinergic (Kunii et al, 2015), and glutamatergic markers (Beneyto and Meador-Woodruff, 2008), as well as abnormalities in various second-messenger systems eg calcium/ calmodulin-dependent protein kinase II-dependent signaling (Robison, 2014). Decreased prefrontal gyrification and dendritic spine density has also been observed in both disorders (Konopaske et al, 2014; McIntosh et al, 2009). The contribution(s) these abnormalities make (if any) to CC deficits in BPD and SZ is an important area for future study as it may help identify pharmacologic mechanisms by which to target these deficits across diseases.

Although BPD and SZ are classified as distinct disorders it is increasingly recognized that the two illnesses share common features in biological and cognitive processes. From a biological point of view, this perspective may not be surprising given the degree of genetic overlap and heritability between the two diseases (Cardno and Owen, 2014; Lee et al, 2013; Pearlson, 2015). Nonetheless, it is important to note that clinically the two diseases have numerous distinctive features (outside of symptoms) such as differences in developmental trajectory, treatment response, and clinical course (Murray et al, 2004). In this study only a single 
dimensional construct was investigated (the goal maintenance function of CC). It is possible (and perhaps highly likely) that along other dimensions with unique behavioral and neural correlates, such as reward processing and emotion regulation, there may be categorical distinctions between the two syndromes. While the present results provide a validation of the approach of examining dimensional measures across diagnostic boundaries and also suggest a continuum of CC dysfunction and its neural underpinnings across BPD and SZ, additional future work examining these and other constructs will be needed to develop a comprehensive understanding of the psychopathology of severe mental illness.

\section{CONCLUSIONS}

In the present study we tested the hypothesis that functional neurocognitive deficits during $\mathrm{CC}$ of goal maintenance transverse traditional diagnostic criteria in BPD and SZ. These results suggest that CC dysfunction may be described as a continuum of deficits across these diseases, supporting an RDoC-based framework of understanding this important aspect of these illnesses.

\section{FUNDING AND DISCLOSURE}

The authors declare no conflicts of interest.

\section{ACKNOWLEDGMENTS}

Undergraduate interns assisted with data collection and processing. Each undergraduate intern contributed to the project for a minimum of 1 year and a maximum of 3 years. A portion of these data were presented at the American College of Neuropsychopharmacology 52nd annual meeting. This study was supported by grant 5R01MH059883 from the National Institutes of Health (Dr Carter).

\section{REFERENCES}

American Psychiatric Association (1997). Practice guideline for the treatment of patients with schizophrenia. Am J Psychiatry 154(4 Suppl): S1-S63.

Andreasen NC (1984a). Scale for the Assessment of Negative Symptoms (SANS). Department of Psychiatry, College of Medicine, The University of Iowa: Iowa City, IA.

Andreasen NC (1984b). Scale for the Assessment of Positive Symptoms (SAPS). Department of Psychiatry, College of Medicine, the University of Iowa: Iowa City, IA.

Arndt S, Andreasen NC, Flaum M, Miller D, Nopoulos P (1995). A longitudinal study of symptom dimensions in schizophrenia. Prediction and patterns of change. Arch Gen Psychiatry 52: 352-360.

Baker JT, Holmes AJ, Masters GA, Yeo BT, Krienen F, Buckner RL et al (2014). Disruption of cortical association networks in schizophrenia and psychotic bipolar disorder. JAMA Psychiatry 71: 109-118.

Barch DM, Berman MG, Engle R, Jones JH, Jonides J, Macdonald A 3rd et al (2009). CNTRICS final task selection: working memory. Schizophr Bull 35: 136-152.

Barch DM, Carter CS, MacDonald AW 3rd, Braver TS, Cohen JD (2003). Context-processing deficits in schizophrenia: diagnostic specificity, 4-week course, and relationships to clinical symptoms. J Abnorm Psychol 112: 132-143.

Beneyto M, Meador-Woodruff JH (2008). Lamina-specific abnormalities of NMDA receptor-associated postsynaptic protein transcripts in the prefrontal cortex in schizophrenia and bipolar disorder. Neuropsychopharmacology 33: 2175-2186.

Bora E, Pantelis C (2015). Meta-analysis of cognitive impairment in first-episode bipolar disorder: comparison with first-episode schizophrenia and healthy controls. Schizophr Bull 41: 1095-1104.

Brambilla P, Macdonald AW 3rd, Sassi RB, Johnson MK, Mallinger AG, Carter CS et al (2007). Context processing performance in bipolar disorder patients. Bipolar Disord 9: 230-237.

Brandt CL, Eichele T, Melle I, Sundet K, Server A, Agartz I et al (2014). Working memory networks and activation patterns in schizophrenia and bipolar disorder: comparison with healthy controls. Br J Psychiatry 204: 290-298.

Brett M, Anton J-L, Valabregue R, Poline J-B (2002). Region of interest analysis using an SPM toolbox [abstract]. Presented at the 8th International Conferance on Functional Mapping of the Human Brain, Sendai, Japan. Available on CD-ROM in NeuroImage, Vol 16, No 2, abstract 497.

Bunge SA, Kahn I, Wallis JD, Miller EK, Wagner AD (2003). Neural circuits subserving the retrieval and maintenance of abstract rules. J Neurophysiol 90: 3419-3428.

Cardno AG, Owen MJ (2014). Genetic relationships between schizophrenia, bipolar disorder, and schizoaffective disorder. Schizophr Bull 40: 504-515.

Carter CS, Minzenberg M, West R, Macdonald A 3rd (2012). CNTRICS imaging biomarker selections: Executive control paradigms. Schizophr Bull 38: 34-42.

Clementz BA, Sweeney JA, Hamm JP, Ivleva EI, Ethridge LE, Pearlson GD et al (2016). Identification of distinct psychosis biotypes using brain-based biomarkers. Am J Psychiatry 173: 373-384.

Cohen JD, Barch DM, Carter C, Servan-Schreiber D (1999). Context-processing deficits in schizophrenia: converging evidence from three theoretically motivated cognitive tasks. J Abnorm Psychol 108: 120-133.

Cole MW, Repovs G, Anticevic A (2014). The frontoparietal control system: a central role in mental health. Neuroscientist 20: 652-664.

Costafreda SG, Fu CH, Picchioni M, Toulopoulou T, McDonald C, Kravariti E et al (2011). Pattern of neural responses to verbal fluency shows diagnostic specificity for schizophrenia and bipolar disorder. BMC Psychiatry 11: 18.

Cross-Disorder Group of the Psychiatric Genomics C, Lee SH, Ripke S, Neale BM, Faraone SV, Purcell SM et al (2013). Genetic relationship between five psychiatric disorders estimated from genome-wide SNPs. Nat Genet 45: 984-994.

Craddock N, O'Donovan MC, Owen MJ (2006). Genes for schizophrenia and bipolar disorder? Implications for psychiatric nosology. Schizophr Bull 32: 9-16.

First MB, Spitzer RL, Gibbon M, Williams JBW (2002). Structured Clinical Interview for DSM-IV-TR Axis I Disorders, Research Version, Patient Edition. Biometrics Research, New York State Psychiatric Institute: New York.

Green MF (2006). Cognitive impairment and functional outcome in schizophrenia and bipolar disorder. J Clin Psychiatry. 67: e12.

Gruber SA, Dahlgren MK, Sagar KA, Gonenc A, Norris L, Cohen $\mathrm{BM}$ et al (2017). Decreased Cingulate Cortex activation during cognitive control processing in bipolar disorder. I Affect Disord 213: 86-95.

Hamilton LS, Altshuler LL, Townsend J, Bookheimer SY, Phillips OR, Fischer J et al (2009). Alterations in functional activation in euthymic bipolar disorder and schizophrenia during a working memory task. Hum Brain Mapp 30: 3958-3969.

Henderson D, Poppe AB, Barch DM, Carter CS, Gold JM, Ragland JD et al (2012). Optimization of a goal maintenance task for use in clinical applications. Schizophr Bull 38: 104-113. 
Hill SK, Reilly JL, Keefe RS, Gold JM, Bishop JR, Gershon ES et al (2013). Neuropsychological impairments in schizophrenia and psychotic bipolar disorder: findings from the Bipolar-Schizophrenia Network on Intermediate Phenotypes (B-SNIP) study. Am J Psychiatry 170: 1275-1284.

Insel T, Cuthbert B, Garvey M, Heinssen R, Pine DS, Quinn K et al (2010). Research domain criteria (RDoC): toward a new classification framework for research on mental disorders. Am J Psychiatry 167: 748-751.

Ivleva EI, Clementz BA, Dutcher AM, Arnold SJ, Jeon-Slaughter H, Aslan S et al (2016). Brain structure biomarkers in the psychosis biotypes: findings from the bipolar-schizophrenia network for intermediate phenotypes. Biol Psychiatry 82: 26-39.

Konopaske GT, Lange N, Coyle JT, Benes FM (2014). Prefrontal cortical dendritic spine pathology in schizophrenia and bipolar disorder. JAMA Psychiatry 71: 1323-1331.

Kunii Y, Zhang W, Xu Q, Hyde TM, McFadden W, Shin JH et al (2015). CHRNA7 and CHRFAM7A mRNAs: co-localized and their expression levels altered in the postmortem dorsolateral prefrontal cortex in major psychiatric disorders. Am J Psychiatry 172: $1122-1130$.

Lee SH, Ripke S, Neale BM, Faraone SV, Purcell SM, Perlis RH et al (2013). Genetic relationship between five psychiatric disorders estimated from genome-wide SNPs. Nat Genet 45: 984-994.

Lesh TA, Niendam TA, Minzenberg MJ, Carter CS (2011). Cognitive control deficits in schizophrenia: mechanisms and meaning. Neuropsychopharmacology 36: 316-338.

Lesh TA, Tanase C, Geib BR, Niendam TA, Yoon JH, Minzenberg MJ et al (2015). A multimodal analysis of antipsychotic effects on brain structure and function in first-episode schizophrenia. JAMA Psychiatry 72: 226-234.

Lesh TA, Westphal AJ, Niendam TA, Yoon JH, Minzenberg MJ, Ragland JD et al (2013). Proactive and reactive cognitive control and dorsolateral prefrontal cortex dysfunction in first episode schizophrenia. Neuroimage Clin 2: 590-599.

Lopez-Garcia P, Lesh TA, Salo T, Barch DM, MacDonald AW 3rd, Gold JM et al (2016). The neural circuitry supporting goal maintenance during cognitive control: a comparison of expectancy AX-CPT and dot probe expectancy paradigms. Cogn Affect Behav Neurosci 16: 164-175.

MacDonald AW 3rd, Cohen JD, Stenger VA, Carter CS (2000). Dissociating the role of the dorsolateral prefrontal and anterior cingulate cortex in cognitive control. Science 288: 1835-1838.

Marengo J, Harrow M, Herbener ES, Sands J (2000). A prospective longitudinal 10-year study of schizophrenia's three major factors and depression. Psychiatry Res 97: 61-77.

McIntosh AM, Moorhead TW, McKirdy J, Hall J, Sussmann JE, Stanfield AC et al (2009). Prefrontal gyral folding and its cognitive correlates in bipolar disorder and schizophrenia. Acta Psychiatr Scand 119: 192-198.

McIntosh AM, Whalley HC, McKirdy J, Hall J, Sussmann JE, Shankar $P$ et al (2008). Prefrontal function and activation in bipolar disorder and schizophrenia. Am J Psychiatry 165: 378-384.

Minzenberg MJ, Laird AR, Thelen S, Carter CS, Glahn DC (2009). Meta-analysis of 41 functional neuroimaging studies of executive function in schizophrenia. Arch Gen Psychiatry 66: 811-822.

Murray RM, Sham P, Van Os J, Zanelli J, Cannon M, McDonald C (2004). A developmental model for similarities and dissimilarities between schizophrenia and bipolar disorder. Schizophr Res 71: 405-416.

Niendam TA, Laird AR, Ray KL, Dean YM, Glahn DC, Carter CS (2012). Meta-analytic evidence for a superordinate cognitive control network subserving diverse executive functions. Cogn Affect Behav Neurosci 12: 241-268.

Niendam TA, Lesh TA, Yoon J, Westphal AJ, Hutchison N, Daniel Ragland J et al (2014). Impaired context processing as a potential marker of psychosis risk state. Psychiatry Res 221: $13-20$.

O'Donoghue S, Holleran L, Cannon DM, McDonald C (2017). Anatomical dysconnectivity in bipolar disorder compared with schizophrenia: a selective review of structural network analyses using diffusion MRI. J Affect Disord 209: 217-228.

Pearlson GD (2015). Etiologic, phenomenologic, and endophenotypic overlap of schizophrenia and bipolar disorder. Annu Rev Clin Psychol. 11: 251-281.

Reichenberg A, Harvey PD (2007). Neuropsychological impairments in schizophrenia: Integration of performance-based and brain imaging findings. Psychol Bull 133: 833-858.

Reilly JL, Hill SK, Gold JM, Keefe RS, Clementz BA, Gershon E et al (2017). Impaired context processing is attributable to global neuropsychological impairment in schizophrenia and psychotic bipolar disorder. Schizophr Bull 43: 397-406.

Robinson LJ, Thompson JM, Gallagher P, Goswami U, Young AH, Ferrier IN et al (2006). A meta-analysis of cognitive deficits in euthymic patients with bipolar disorder. J Affect Disord 93: 105-115.

Robison AJ (2014). Emerging role of CaMKII in neuropsychiatric disease. Trends Neurosci 37: 653-662.

Rougier NP, Noelle DC, Braver TS, Cohen JD, O'Reilly RC (2005). Prefrontal cortex and flexible cognitive control: rules without symbols. Proc Natl Acad Sci USA 102: 7338-7343.

Sheffield JM, Kandala S, Tamminga CA, Pearlson GD, Keshavan MS, Sweeney JA et al (2017). Transdiagnostic associations between functional brain network integrity and cognition. JAMA Psychiatry 74: 605-613.

Stahl SM (2014). Prescriber's Guide: Stahl's Essential Psychopharmacology5 edn.Cambridge University Press: Cambridge, U.K.. p 824.

Strakowski SM, Eliassen JC, Lamy M, Cerullo MA, Allendorfer JB, Madore $\mathrm{M}$ et al (2011). Functional magnetic resonance imaging brain activation in bipolar mania: evidence for disruption of the ventrolateral prefrontal-amygdala emotional pathway. Biol Psychiatry 69: 381-388.

Tamminga CA, Pearlson G, Keshavan M, Sweeney J, Clementz B, Thaker G (2014). Bipolar and schizophrenia network for intermediate phenotypes: outcomes across the psychosis continuum. Schizophr Bull 40(Suppl 2): S131-S137.

Ventura J, Lukoff D, Nuechterlein KH, Liberman RP, Green MF, Shaner A (1993). Manual for the expanded brief psychiatric rating scale. Int J Methods Psychiatric Res 3: 227-244.

Volk DW, Sampson AR, Zhang Y, Edelson JR, Lewis DA (2016). Cortical GABA markers identify a molecular subtype of psychotic and bipolar disorders. Psychol Med 46: 2501-2512.

Weschler D (1999). Weschler Abbreviated Scale of Intelligence (WASI). Harcourt Assessment: San Antonio, TX.

Whalley HC, Papmeyer M, Sprooten E, Lawrie SM, Sussmann JE, McIntosh AM (2012). Review of functional magnetic resonance imaging studies comparing bipolar disorder and schizophrenia. Bipolar Disord 14: 411-431.

Woods SW (2003). Chlorpromazine equivalent doses for the newer atypical antipsychotics. J Clin Psychiatry 64: 663-667.

Yoon JH, Minzenberg MJ, Ursu S, Ryan Walter BS, Wendelken C, Ragland JD et al (2008). Association of dorsolateral prefrontal cortex dysfunction with disrupted coordinated brain activity in schizophrenia: relationship with impaired cognition, behavioral disorganization, and global function. Am J Psychiatry 165: 1006-1014. 